\title{
Grosse Persönlichkeit, gepaart mit respektvoller Bescheidenheit
}

\section{Edouard Battegay}

Direktor Klinik und Poliklinik für Innere Medizin, UniversitätsSpital Zürich
Korrespondenz:

Prof. Dr. med. Edouard Battegay Klinik und Poliklinik

für Innere Medizin

UniversitätsSpital

Rämistrasse 100

CH-8091 Zürich
Sehr geehrter Herr Dekan, lieber Klaus, lieber Felix

Heute würdigen und verabschieden wir Dich, Felix, was eigentlich kaum vorstellbar ist. Denn Du bist voller Tat- und Schaffenskraft, und ich bzw. wir gehen davon aus, dass die «Verabschiedung» für Dich eigentlich einen Schub an neuen Aktivitäten im erweiterten beruflichen Umfeld bedeutet.

Mehr als 25 Jahre hast Du als Direktor das 1963 gegründete Institut für Sozial- und Präventivmedizin der Universität Zürich mit sicherer Hand geführt und warst beliebter ordentlicher Professor der Universität und zudem ein sehr ordentlicher und beliebter Chef. (Dein Institut hat während seiner Existenz damit nun erst zwei Chefs gehabt, Meinrad Schär und Dich.) Vor Deinem Wechsel nach Zürich warst Du schon 5 Jahre als ordentlicher Professor und Leiter des gleichnamigen Institutes der Universität Lausanne tätig. 30 Jahre als ordentlicher Professor in Sozial- und Präventivmedizin! In unserer universitären Welt ist das eigentlich eine biblische Zeit, eine methusalemartige Zahl. Zudem hast Du über 10 Jahre einen Lehrauftrag an der ETH (Humanernährung) und Dein Fach während 9 Jahren als Forschungsrat des Nationalfonds vertreten (Abt. Medizin und Biologie, 1991-2000).

Aber nochmals zurück zum Amtsbeginn am Institut für Sozial- und Präventivmedizin vor mehr als 25 Jahren: Du fandest am Institut ungefähr 15 Mitarbeitende und, immerhin, 2 Computer vor. Heute sind es gegen 10-mal mehr Mitarbeitende (nämlich rund 140, verteilt auf 194 Anstellungen) sowie ein Budget von rund 15 Millionen, das zu rund 40\% über Drittmittel finanziert wird. Aus den beiden Computern ist die Abteilung Biostatistik mit Prof. Leo Held an der Spitze geworden. Du übergibst Deinem Nachfolger ein Institut, ja eine Institution mit sehr grosser Strahlkraft, Deine Strahlkraft war und ist kontagiös, aber ich greife vor.

Du hast in Deinen Konzepten immer die grosse Klammer, die grossen Zusammenhänge zu verstehen, zu formen und zu verbessern gesucht. Entsprechend ist diese Laudatio angelegt.

Ich möchte das berufliche Leben von Prof. Felix Gutzwiller in drei Abschnitte teilen, nämlich

- Von Gesellschaft zu Prävention

- Von Prävention zu Politik

- Von Politik zu Gesellschaft

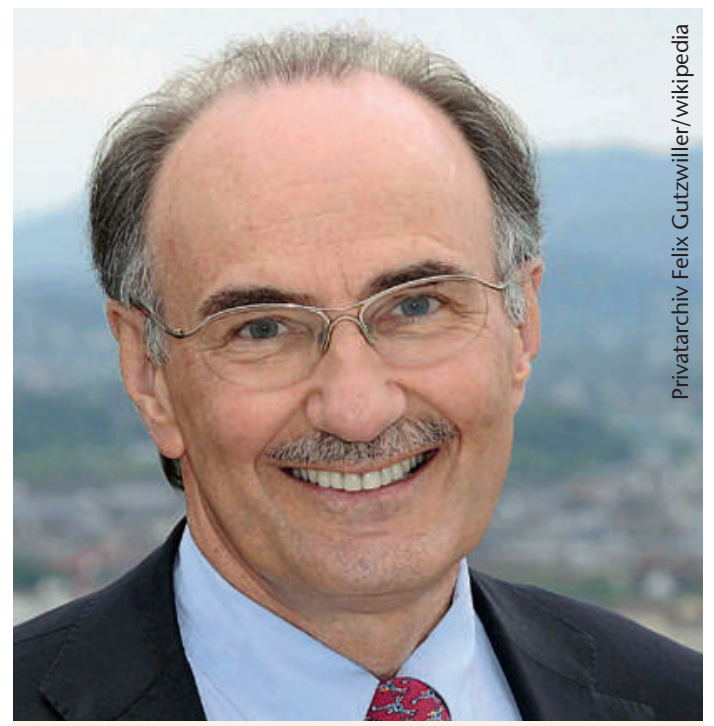

Prof. Dr. med. Felix Gutzwiller leitete mehr als 25 Jahre das Institut für Sozial- und Präventivmedizin der Universität Zürich

\section{Von Gesellschaft zu Prävention}

Felix Gutzwiller ist in Basel in einem ausgeprägt humanistischen Umfeld aufgewachsen. Zu Hause und in der Schule (die Begriffe zu Hause und Schule waren in Felix' Fall teilweise synonym, weil Felix' Vater verehrter Rektor des Humanistischen Gymnasiums war) suchte man das Verständnis der grossen Zusammenhänge von Geschichte und Gesellschaft. Deshalb ist es wohl nicht zufällig, dass Felix Gutzwiller nach seinem Medizinstudium in Basel einen Master in Public Health in Harvard sowie ein Doktorat in Public Health an der Johns Hopkins University erlangte.

Felix Gutzwiller wollte Gesundheit und Krankheit im grossen gesellschaftlichen Kontext aktiv angehen.

Mit dem ersten nationalen Forschungsprogramm mit dem Titel «Prävention von Herz-Kreislauf-Krankheiten in der Schweiz» kehrte Felix nach Basel an die Abteilung für Kardiologie zurück, deren damaliger Leiter Prof. Walter Schweizer Präsident der Expertengruppe dieses Programmes war. So wurde die erste Gemeinde-Interventionsstudie in der Schweiz mit ganzen Gemeinden als statistischen Einheiten durchgeführt, nach wie vor nicht nachgeahmt in unserem 
Land. Diese und andere Studien waren der Ausgangspunkt für eine hervorragende wissenschaftliche Tätigkeit im Bereich der Herz-Kreislauf-Epidemiologie und Prävention. Felix führte auch internationale Studien durch, zu erwähnen wäre insbesondere die grosse MONICA-Studie Monitoring of trends and determinants of Cardiovascular Diseases (koordiniert von der Weltgesundheitsorganisation WHO): Diese riesige internationale epidemiologische Landmarkstudie wurde in den Jahren 1990/1991 von Felix Gutzwiller geleitet.

\section{Von Prävention zu Politik}

Felix hat neben der Herz-Kreislauf-Epidemiologie schon früh das Interesse für die Zusammenhänge im Gesundheitswesen gepackt. In den USA hatte er erfahren, wie sehr das Gesundheitswesen selbst Gegenstand wissenschaftlicher Untersuchungen sein kann. So engagierte er sich gestaltend auch an dieser Front und leitete im Rahmen eines weiteren nationalen Forschungsprogrammes (Nr. 8 «Wirtschaftlichkeit und Wirksamkeit im schweizerischen Gesundheitswesen») den ersten nationalen Gesundheitssurvey und publizierte (zusammen mit Prof. Li Zemp) den ersten schweizerischen Frauengesundheitsbericht.

Mit dem Aufkommen von HIV/Aids und der Drogenproblematik in der Schweiz begann Felix eine dritte Thematik zu beschäftigen und zu faszinieren. Es musste und konnte etwas gemacht werden, um die Problematik in den Griff zu bekommen. So hat er in Zusammenarbeit mit dem Bundesamt für Gesundheit (Dr. Bertino Somaini) die Stop Aids-Kampagnen aufgebaut sowie deren Evaluation vorangetrieben. Zusammen mit Prof. Ambros Uchtenhagen wurden die umfangreichen Zürcher Studien zur Heroinsubstitution bei schwer Abhängigen durchgeführt.

Prof. Felix Gutzwiller hat also ein ausserordentlich breites und unternehmerisch zum Erfolg geführtes Interessenspektrum. Kein Zufall also, dass er in nationale und internationale Beratungsgremien und Expertenkommissionen, aber auch Gesundheitsorganisationen berufen wurde.

Für Prof. Felix Gutzwiller hat Public Health, die Gesundheit ganzer Bevölkerungen, immer zwei Pole, die auch der Philosophie und Strategie seines Institutes zugrunde liegen, nämlich Wissenschaft und Aktion-Intervention. Public Health muss zwingendermassen auch politische Intervention und Gestaltung sein. Es überrascht deshalb nicht, dass sich Felix zunehmend auch für die nationale Politik engagierte: ab 1999 im Nationalrat, ab 2007 im Ständerat für den Kanton Zürich. Ein wohl bleibendes Beispiel für diese Synergien zwischen Politik und evidenzbasierter Epidemiologie ist der Einsatz von Felix Gutzwiller für rauchfreie öffentliche Räume. Dass es dazu heute in der Schweiz eine gesetzliche Grundlage gibt, ist auf seinen entsprechenden parlamentarischen Vorstoss im Jahre 2004 zurückzuführen.

\section{Von Politik zu Gesellschaft}

Felix wird sich in diesen Funktionen in Politik und strategischen Gremien weiterhin auch für die schweizerischen Hochschulen und die Universität Zürich einsetzen können, als Mitglied beispielsweise der Kommission für Soziales und Gesundheit SGK, vor allem aber auch als Präsident der Kommission für Wissenschaft, Bildung und Kultur WBK des Ständerates. Auch seinem akademischen Kerngebiet bleibt er verbunden, so etwa als Präsident des Kuratoriums des Swiss Tropical und Public Health Institutes (Swiss TPH) in Basel. Felix, Du wirst noch lange an der Zukunft der Schweiz mitgestalten, das ist mir und allen hier im Saal sonnenklar.

Diese beeindruckenden Leistungsausweise wären ohne hervorragende Persönlichkeitsmerkmale von Dir kaum vorstellbar:

- Arbeitskraft und Ausstrahlung

- Optimismus und Offenheit

- Förderung und Freiheit

Arbeitskraft und Ausstrahlung: Ohne Fleiss und Arbeitskraft, aber doch Gelassenheit, wäre ein Werdegang wie der Deine nicht möglich. Auch dank einem augenzwinkernden, auch selbstkritischen Humor kommt nie der Eindruck von Verbissenheit auf. Du hast ein hohes Mass an Durchsetzungsvermögen. Aber ohne persönliche Ausstrahlung hätte sich die Arbeitskraft auf Dein Umfeld, und das ist in der Zwischenzeit wie geschildert extrem gross, kaum so befruchtend ausgewirkt.

Optimismus und Offenheit: Du bist immer optimistisch, identifizierst früh aus einer breiten Perspektive das Potential für Verbesserung mit scharfem Verstand und packst visionär Chancen für Aufgaben, Institutionen, Gesellschaft und den Aufbau der Zukunft.

Förderung und Freiheit: Du hast viele und vieles mit ausserordentlichem Geschick und unternehmerisch gefördert, aber gleichzeitig jedem so viel Freiheit wie möglich gelassen, dies durfte ich auch selber erleben. Das ist eine ganz ausserordentliche Leistung. Und für jede und jeden um Dich herum waren jederzeit ganz stark Werte, tiefverankert in einer generationenumspannenden humanistischen westlichen Kultur, zu spüren gewesen. Die grosse Persönlichkeit, gepaart mit respektvoller Bescheidenheit. Für alles sind wir, die Medizinische Fakultät und die Universität Zürich, Dir ausserordentlich dankbar.

Wir wünschen Dir, dass diese von Dir ausgehende Kraft, Schaffenskraft und Strahlkraft, weiterhin zugunsten der Gesellschaft und Zukunft wirken möge, auch zugunsten der Universität Zürich und deren Institutionen. Dazu und auch sonst wünschen wir Dir und den Deinen von Herzen Gesundheit und Glück. Danke! 